\title{
El desafío de la carrera de Licenciatura en Enfermería frente a las demandas sociales
}

\section{Verónica Valeria Cabrera}

Docente del Instituto Universitario

de la Policía Federal, Argentina

vale.vero_cabrera@hotmail.com

Lorena Guadalupe Ciccarelli

Docente del Instituto Universitario

de la Policía Federal.

lorecicca78@gmail.com
Silvio Damián Saravia

Docente de la Universidad Nacional de Tres de Febrero, Buenos Aires,

Argentina.

silviosaravia@gmail.com
Integración de la docencia y la extensión /

Desafíos de gestión

RECEPCIÓN: 24/06/16

ACEPTACIÓN FINAL: 13/10/16

\section{Resumen}

La universidad, desde sus comienzos, es reconocida como institución de primer orden en la transferencia del conocimiento científico y profesional. Se entiende que es un agente fundamental de la innovación atravesada por el contexto sociopolítico y económico que da respuestas a las necesidades de los habitantes. Para mantenerse a la par de las aceleradas reformas y cambios que se producen en la comunidad y en el ejercicio de la profesión, la formación en la carrera de Licenciatura en Enfermería debe gestionar cambios curriculares significativos, como en el área del conocimiento, producción, organización y divulgación. Cuestiones que se plantean al momento del proceso de acreditación por parte de la Comisión Nacional de Evaluación y Acreditación Universitaria (CONEAU). En este artículo se reflexionará sobre los desafíos que tiene la carrera de Licenciatura en Enfermería para lograr la integración de los tres principales pilares de la universidad (docencia, investigación y extensión universitaria).

Palabras-clave

- Estrategia

- Desafío en Enfermería

- Didáctica

\section{Resumo}

A Universidade desde a sua criação é reconhecido como uma instituição de primeira ordem na transferência de conhecimento científico e profissional. Entende-se que é um agente fundamental da inovação atravessada pelo contexto sociopolítico e econômico, que da respostas às necessidades dos habitantes. Para manter a sincronia com reformas aceleradas e as mudanças que ocorrem na comunidade e no exercício da profissão, a formação no curso da Escola de Enfermagem deve gerenciar mudanças curriculares significativas, como na área do conhecimento, da produção, da organização e da divulgação. Questões que se expõem no momento do processo de acreditação pela Comissão Nacional de Avaliação e Acreditação Universitária (CONEAU).

Neste artigo se refletirá sobre os desafios que tem o curso do Bacharelado em Enfermagem para conseguir a integração dos três pilares principais da universidade (docência, pesquisa e extensão universitária).

Palavras-chave

- Estratégia

- Desafio em Enfermagem

- Didática 


\section{Introducción}

La integración de la docencia, la investigación y la extensión como componente del currículo universitario es uno de los pilares necesarios para la producción de nuevo conocimiento y la búsqueda de alternativas para solucionar temas vinculados a la salud de la población.

A nivel mundial se observa, principalmente en las últimas décadas, un estudio constante sobre nuevas formas de organización académica que permitan brindar una mejor respuesta a los requerimientos de la sociedad. El convencimiento de que no es posible resolver los complejos problemas de la sociedad sin una perspectiva interdisciplinaria hace que el meollo de las reformas académicas de nuestros días radique en cómo combinar los elementos estructurales de la universidad de manera que su organización promueva y facilite esa interdisciplinariedad, que es la forma contemporánea de ejercer el oficio universitario. En la misma línea que Egurrola (2005), se puede decir que el problema no se reduce a la relación puntual entre docencia e investigación. Se trata de cómo la universidad utiliza estas funciones orientándolas al desarrollo de nuevo conocimiento y de cómo alumnos, docentes e investigadores la transfieren a la sociedad. De allí que se considere necesaria la construcción de un modelo didáctico en el que no solo se pueda integrar la docencia y la investigación para la producción del conocimiento sino que, además, ese conocimiento pueda ser proyectado en ámbitos comunitarios en donde sea requerido.

Es decir, posibilitar la extensión del conocimiento producido en las universidades a la sociedad.

Es un desafío que nos invita ciertamente a la búsqueda creativa y urgente tanto de posibilidades de intercambio entre cada una de esas funciones universitarias como de espacios de convergencia donde esas actividades logren armonizarse como partes de un todo. Que la investigación esté contenida en la docencia y la docencia en la investigación y ésta deje un requisito o formalidad académica (tesina de grado) para optar a un título universitario.
De esta manera, se apuesta a que se conjugue el hacer científico con los procesos de enseñar y aprender, aplicando ese conocimiento producto de la integración de ambas funciones a través de la extensión universitaria.

En la actualidad, es ineludible que la universidad resuelva los requerimientos que la sociedad le plantea a través de un proceso formativo de los futuros Licenciados en Enfermería, que permita generar en los profesionales una disposición abierta para incorporar y construir nuevos saberes acerca de este mundo cultural y axiológico que no cesa de transformarse (Capella, 2002). Por ello es necesario desarrollar estrategias para fortalecer la capacidad investigativa del estudiante fundamentadas en una relación caracterizada por el diálogo crítico, en el que se incorpore la lógica de la investigación científica en el proceso docente educativo.

Para ser capaz de generar las transformaciones deseadas en la sociedad mediante los profesionales en formación, los que sabrán valorar esas necesidades en cada localidad considerando el contexto nacional y mundial. Se entiende que, en la medida en que se producen las demandas sociales como necesidades sentidas, así también en el currículo se deben producir transformaciones deseables para responder a ellas.

En este sentido, el desarrollo curricular en los espacios de aprendizaje universitarios desde la perspectiva del modelo propuesto deberá adecuarse a una nueva dinámica didáctica basada en procesos complejos, sistémicos e integrados a los cambios paradigmáticos emergentes que responden a una sinergia de las tres áreas de la universidad (docencia, investigación y extensión), en donde se logre formar profesionales con compromiso social.

\section{La formación de profesionales con una nueva metodología} Es necesario reivindicar la complejidad de la relación entre la docencia y la investigación dado que la ésta es un elemento 


\section{4}

\section{en el caso de Enfermería continúa \\ siendo escasa la producción y especialmente la transferencia de lo investigado}

esencial de la institución universitaria. Ciertamente, para que una institución se precie de ser una universidad es preciso que se apoye en la investigación -como actividad generadora o creadora de conocimientos_ para fundamentar su quehacer académico, para hacer posible una formación profesional adecuada y una docencia de alta calidad en permanente enriquecimiento. La búsqueda del equilibrio entre las funciones básicas de docencia y la investigación para su posterior transferencia es uno de los retos que debe enfrentar la educación superior y que sólo se resuelve si todas las funciones contribuyen al logro de su misión educativa: formar académicos profesionales y especialistas dotados del saber y las destrezas adecuadas y a la vez contribuir al adelanto, ampliación y difusión del conocimiento.

Para lograr que los Licenciados en Enfermería posean un compromiso social mayor desde los primeros años de su formación, con participación activa en trabajos de investigación sobre problemáticas de la sociedad que los rodea, se requiere tener profesores igualmente bien formados no sólo en cuestiones didácticas y pedagógicas, sino en investigación y extensión universitaria. Por eso es fundamental la integración que preparará a largo plazo profesionales idóneos en su disciplina pero con una orientación hacia la investigación. Claro está que también serán necesarias políticas universitarias que permitan acceder a concursos docentes con dedicación exclusiva, para que el formador de profesionales cuente con tiempo y recursos para la dedicación a la investigación.

Al respecto, la Conferencia sobre Políticas y Estrategias para la Transformación de la Educación Superior en América Latina y el Caribe, que presidió la Conferencia Mundial de Educación Superior convocada por la UNESCO, declaró que: "los objetivos de la Educación Superior pueden resumirse en unos pocos conceptos, que encierran un mundo de gran complejidad: formar ciudadanos responsables y comprometidos; proporcionar los profesionales que la sociedad necesita; desarrollar la investigación científica y técnica; conservar y transmitir la cultura; actuar como memoria del pasado y atalaya del futuro y constituir una instancia crítica, basada en el rigor y el mérito, que puede ser, por todo ello, vanguardia, a todas las escalas de la solidaridad intelectual y moral". (1998:8)

Por lo que se entiende, es imprescindible la investigación para poder acceder al conocimiento de las necesidades sociales y darles respuestas. Y allí la extensión universitaria es el eje principal para volcar los contenidos originados y llevarlos a la práctica en la sociedad.

Actualmente, la mayoría de las carreras de las universidades públicas realiza trabajos de investigación, y en el caso de Enfermería continúa siendo escasa la producción y especialmente la transferencia de lo investigado. Solo basta con observar las tesis de grado de los Licenciados en Enfermería para darse cuenta de que temas innovadores de investigaciones de egresados descansan en las bibliotecas de las universidades, y muchas veces ese conocimiento obtenido podría mejorar la calidad de vida o las demandas de la sociedad.

La preparación de los Licenciados en Enfermería como ente activo en la sociedad en la que se desempeñan exige madurez y capacidad para evaluar, sintetizar rápida y correctamente un gran volumen de información, vinculando la teoría con la práctica, 
con carácter científico y pensamiento crítico de los contenidos para promover que las aptitudes y la resolución de problemas sean culturalmente apropiados. Los fundamentos del nuevo modelo traen consigo la modificación de los perfiles de salud, dan respuesta social a través de la investigación y logran con la docencia la reproducción de la información obtenida para que, a través de la extensión, se logren acciones que respondan a las problemáticas encontradas y estudiadas.

De esta forma se crea un escenario particular donde las proyecciones locales de salud pueden tener la correspondiente respuesta académica a corto o mediano plazo; se favorecen las condiciones de una interacción verdadera entre las instituciones universitarias y la comunidad e infinidad de proyectos expresan realidades concretas de determinadas personas en condiciones específicas.

El eje principal durante toda la carrera de Licenciado en Enfermería debería ser la búsqueda de problemáticas de la sociedad para que, a partir de ello, se realicen actividades para la comunidad, en la comunidad y con la comunidad.

\section{La Responsabilidad Social Universitaria (RSU)} y las funciones de docencia, investigación y extensión El cumplimiento de la RSU implica generar impactos que favorezcan al entorno local en donde la universidad se encuentra inmersa, y esto es posible a través del cumplimiento exitoso de las funciones fundamentales de las universidades, como lo son la docencia, la extensión y la investigación. Por lo que en la gestión institucional universitaria mucho se ha dicho con respecto a la llamada articulación de estas tres funciones y en la formalidad de normatividad existente se han realizado marcados esfuerzos para lograrlo, sin embargo solo se vinculan estrechamente en ese nivel. La integración de la ejecución curricular didáctica en los espacios universitarios es una práctica que producirá grandes cambios y mejorará la participación de los futuros profesionales de la salud comprometidos con la sociedad.

Debido a la necesidad cada día más sentida de que los currículos oficiales den respuesta pertinente a las demandas sociales, es preciso enfatizar entonces que los elementos sustantivos de la realidad actual platean la posibilidad a desarrollar, por parte del docente universitario, sus propias prácticas y sus propias teorías del currículo.

En este sentido, la docencia, la investigación y la extensión como construcción del conocimiento en los espacios de aprendizaje son vistas cada una como parte de la otra sin reducirse jamás una a la otra, superando el pensamiento unidimensional y fragmentario con un nuevo modo de pensamiento que integra lo uno y lo múltiple de cada una en la totalidad globalizante de ambos, en tanto esta perspectiva constituye un avance epistemológico importante en el conocimiento de los complejos procesos sociales.
La reflexión sobre las prácticas docentes universitarias ha consistido en la búsqueda de estrategias y procedimientos que lleven a los profesores a resolver los problemas que imponen el fenómeno de la masificación y la explosión del conocimiento en los diferentes campos disciplinarios.

Hay un punto nodal en la profesionalización de la docencia como una de las categorías sustantivas de la práctica educativa y la vinculación docencia-investigación como la alternativa idónea para conseguirlo. La docencia y la investigación son funciones universitarias íntimamente vinculadas, no hay docencia de calidad que no se apoye en resultados de investigación, y es precisamente la docencia el espacio natural para comunicar, analizar y discutir sus resultados. Este vínculo se fortalece y cobra mayor significación al considerar a la docencia como objeto de investigación del docente, a éste como investigador de su propia práctica educativa y con un compromiso social universitario que, mediante las actividades, acreciente la relación la sociedad y la comunidad académica.

\section{Conclusiones}

Se plantea una mayor articulación e integración de tres funciones básicas de la universidad, lo que no solo es una idea que en la actualidad conserva su plena vigencia, sino que es una necesidad a la cual estamos obligados a dedicar mayor atención.

Aunar la integración de la docencia y la investigación propiciará la producción y aplicación del conocimiento de los espacios de aprendizaje universitarios a la sociedad como una sola función para la extensión y socialización de dicho conocimiento.

Este modelo permitirá viabilizar la combinación de las funciones universitarias a nivel didáctico y con gran complementariedad, para que su desarrollo no sea desde lógicas distintas sino que esas lógicas compartan una concepción de y ante el conocimiento. Para ello será necesario el fomento de una cultura de investigación en el quehacer de la educación.

Para la universidad de hoy y la del mañana resulta necesario trabajar para crear estructuras académicas con medios intelectuales y materiales que posibiliten la producción del conocimiento extramuros, construcción de saberes teóricos que sustenten la investigación científica en el campo pedagógico, en el marco de la reflexión y análisis de los fundamentos —-filosóficos, sociológicos, pedagógicos y psicológicos- de la educación superior. La extensión universitaria posee una función esencial de la actividad universitaria, puesto que se asume como un proceso formativo, dinámico, integrador, multifacético en la comunidad y para la comunidad respaldado por un sistema de formación, capacitación, estimulación y comunicación. Aspectos importantes para la formación de Licenciados en Enfermería que permitirán a los futuros profesionales, actores de la sociedad, responder a las necesidades emergentes con visión de presente y futuro. 


\section{4}

planteamos la necesidad de que

la formación de los Licenciados

en Enfermería responda

activamente a las necesidades

sociales y que no solo tengan

un acercamiento a la sociedad

en un determinado período

durante su formación práctica
La actuación en el campo social es el eje central de la labor extensionista, coadyuva a elevar el nivel de conciencia y la capacidad de acción, tanto individual como colectiva, a través de sus proyectos en vertientes fundamentales en la comunidad universitaria y extrauniversitaria, con la identificación de las principales necesidades de salud según el análisis de la situación. El valor radica en propiciar el espacio y las herramientas necesarias para llevar estos proyectos a hechos, potenciando la participación y el protagonismo de los actores sociales.

Pero para que lo propuesto sea posible se requiere de una reflexión profunda en la que participen todos los actores de la universidad (estudiantes, docentes-investigadores, consejo académico y departamentos de extensión e investigación y desarrollo), como también de municipios y organismos públicos que apoyen las iniciativas de acercar más a los profesionales en formación a las necesidades de la población que los rodea, pues el gran aporte a la sociedad desde la universidad sólo se logrará mediante actividades en conjunto.

Si bien en varias universidades existen tareas de extensión, gran parte de ellas no responde en su totalidad a necesidades sociales. Lo que se propone es que las actividades de extensión sean el producto de los datos recabados en las tesis de grado o actividades de investigación que realizan docentes y alumnos de una misma carrera o en conjunto con otras.

Planteamos la necesidad de que la formación de los Licenciados en Enfermería responda activamente a las necesidades sociales y que no solo tengan un acercamiento a la sociedad en un determinado período durante su formación práctica. Que existan actividades que involucren la docencia, la investigación y la extensión durante toda su formación para lograr dar más y mejores respuestas a las demandas sociales en las áreas en que se vea involucrada la Enfermería y que se activen mecanismos de coparticipación con organismos locales y nacionales para lograr esas metas.

\section{Referencias bibliográficas}

Capella J. (2002). Análisis de la educación desde la perspectiva local: condicionamientos, oportunidades y consolidación de la identidad. En Hünermann, P.; Neuser, H.; Pérez Valera, V.M., Formar, educar, aprender: promoción humana integral en una cultura global adveniente. Buenos Aires.

Egurrola, J. (2005). La interacción investigación y docencia. Sustento para el desarrollo académico de las unidades multidisciplinarias de la Universidad Nacional Autónoma de México. México: Plaza y Valdés.

UNESCO (1998). Conferencia Mundial sobre la Educación Superior. La educación superior en el siglo XXI. Visión y acción. París, 5-9 de octubre. Recuperado de http:// www.unesco.org/education/educprog/wche/declaration_spa.htm (18/06/2016). 